manage risk alone. They form an integral part of a coordinated and collaborative effort between civil society, the private sector and government that works best where there are established structures and systems in place, and partnerships built on trust that have been forged before a crisis strikes.

Methods: Case study.

Results: Communities are central to epidemic and pandemic preparedness.

Conclusion: Global and national public health expertise must recognize this role and work toward how engagement with communities and civil society, can become central to their preparedness and response efforts. Through the presentation of several case studies from the Red Cross and Red Crescent movement, best practice and opportunities for improvement will be showcased. Case studies from a variety of contexts will show how it is possible to include joint planning and implementation, moving beyond risk communication to effective two-way participation, ensuring public health response is understood by, and designed for, the communities they serve in acute and recovery phases.

Prehosp Disaster Med 2017;32(Suppl. 1):s205-s206

doi:10.1017/S1049023X17005362

The National Health Security Strategy and Implementation Plan: An Overview

\section{Charlotte Spires}

Assistant Secretary for Preparedness and Response, US Dept. Health and Human Services, District of Columbia/United States of America

Study/Objective: The purpose of this presentation is to provide an overview of the US Department of Health and Human Services' National Health Security Strategy (NHSS). The NHSS is a congressional mandate to achieve a health-secure and resilient nation by minimizing the health consequences of large-scale emergencies. The NHSS Implementation Plan (IP) which elaborates on activities that stakeholders might undertake to address the priorities of the NHSS will also be discussed.

Background: The 2015-2018 NHSS is a national strategy that envisions a nation that is secure and resilient in the face of diverse incidents with health consequences. The goal of the NHSS is to strengthen and sustain communities' abilities to prevent, protect against, mitigate the effects of, respond to, and recover from incidents with negative health consequences. The IP serves as a framework to help guide the nation and facilitate collaboration and coordination among stakeholders to advance national health security.

Methods: The presentation will provide an overview of the NHSS and the accompanying IP. The five objectives of the NHSS include: (1) build and sustain healthy, resilient communities; (2) enhance the national capability to produce and effectively use both medical countermeasures and nonpharmaceutical interventions; (3) ensure comprehensive health situational awareness to support decision making before incidents and during response and recovery operations; (4) enhance the integration and effectiveness of the public health, health care, and emergency management systems; and (5) strengthen global health security.
Results: Strategic outreach and engagement like this will play a major role in socializing national health security and motivating stakeholders to take actions that address NHSS objectives.

Conclusion: Achieving the goal of preparing for, and responding to, large-scale health consequences will require a willingness to engage a broad array of stakeholders in an ongoing collective ability to recognize, confront, and resolve existing and emerging threats to domestic health.

Prehosp Disaster Med 2017;32(Suppl. 1):s206

doi:10.1017/S1049023X17005374

Feasibility of the Novel Combination of Influenza Vaccinations and Child Passenger Safety Seat Fittings in the Drive-Thru Clinic Setting

Ngoc A. Le, Rachel Charney, James Gerard

Pediatrics, Saint Louis University/SSM Cardinal Glennon Children's Hospital, Saint Louis/MO/United States of America

Study/Objective: We hypothesized that combining influenza vaccinations and child passenger seat fittings (CPSF) in a drivethru clinic (DTC) format will be both feasible and desired by the community.

Background: Disaster medicine is an ever-evolving area of medicine with the purpose of helping the masses quickly and efficiently. The drive-thru clinic (DTC) model is a disaster tool that allows distribution of supplies or services while participants remain in their automobiles. Influenza vaccination is the most commonly utilized form of the DTC and has been utilized in metropolitan areas successfully as a single service.

Methods: Each automobile's driver was verbally surveyed at each station of the DTC. The survey content involved satisfaction and background health habits.

Results: In our inaugural combined service, five hour-long DTC there were 86 automobiles served that contained 161 children, of which 28 also participated in CPSF. Each CPSF station required one extra worker in comparison to the traditional DTC influenza model. The median total clinic time was 9:00 (IQR 6:00, 14:00) minutes. For those who only received influenza vaccines, the median total clinic time was 7:30 (IQR 6:00, 10:00) minutes. For those who received both services, the median total clinic time was 27:00 (IQR 22:20, 33:30) minutes with an average of 1.75 CPSFs per automobile.

Conclusion: This was a pilot study involving multiple services in the DTC model and is the first of its kind in the literature. Our clinic was successful in executing both services without sacrificing speed, convenience, or patient satisfaction. Additional studies are needed to further evaluate the efficacy of the multiple service DTC.

Prehosp Disaster Med 2017;32(Suppl. 1):s206

doi:10.1017/S1049023X17005386

Epidemiology of Poisoning Patients Presenting to the Emergency Center of Princess Marina Hospital in

Gaborone, Botswana

Pon-Hsiu Yeh ${ }^{1}$, Eric Cioe ${ }^{2}$, Aurelio Rodriguez ${ }^{3}$, Megan Cox ${ }^{4}$

1. Emergency Department, New York Presbyterian Hospital, New York/AL/United States of America 